\title{
Un espace métrique linéaire qui n'est pas un rétracte absolu
}

par

\author{
Robert Cauty (Paris)
}

\begin{abstract}
We construct the example of the title.
\end{abstract}
1. Introduction. Le but de cet article est de démontrer le théorème suivant, qui résout un problème classique.

1.1. ThÉORÈME. Il existe un espace métrique linéaire $\sigma$-compact $E$ qui n'est pas un rétracte absolu.

Il est connu qu'alors le complété de $E$ ne peut pas être un rétracte absolu. Cet espace, donc son complété, possède aussi la propriété intéressante suivante :

1.2. AdDendum. E peut être construit de façon à être un sous-espace vectoriel fermé d'un espace métrique linéaire qui est un rétracte absolu.

$E$ fournit aussi le premier exemple d'espace métrique linéaire à ne pas être admissible au sens de Klee [11] puisque Dobrowolski a prouvé dans [4] qu'un espace métrique linéaire $\sigma$-compact est admissible au sens de Klee si, et seulement si, c'est un rétracte absolu.

Notre construction utilise l'espace vectoriel topologique libre $E(X)$ sur un compact métrisable $X$. Algébriquement, $E(X)$ a pour base $X$, et sa topologie est la plus fine des topologies vectorielles induisant sur $X$ sa topologie originelle. $E(X)$ n'est pas métrisable, mais sa topologie est la borne supérieure de l'ensemble $\mathcal{T}(X)$ des topologies vectorielles métrisables qui induisent sur $X$ sa topologie de départ; plus précisément, pour tout ouvert $U$ de $E(X)$, il existe $\tau \in \mathcal{T}(X)$ telle que $U$ soit $\tau$-ouvert. Bien que nous la présentions un peu différemment dans la suite, notre stratégie est la suivante. Il est connu qu'un espace métrisable $Y$ est un rétracte absolu de voisinage si, et seulement si, tout ouvert de $Y$ a le type d'homotopie d'un

1991 Mathematics Subject Classification: Primary 54C55. 
CW-complexe (voir [3]). Si $\tau$ appartient à $\mathcal{T}(X)$ et si le sous-ensemble $U$ de $E(X)$ est $\tau$-ouvert, l'identité $i$ est une équivalence homotopique faible de $U$ dans $(U, \tau)$. Si $(E(X), \tau)$ est un rétracte absolu, il est facile de voir que $i$ a un inverse homotopique à droite, i.e. que $U$ est dominé par $(U, \tau)$, donc $U$ doit alors, comme $(U, \tau)$, avoir le type d'homotopie d'un CW-complexe, la fonction $i$ étant alors en fait une équivalence homotopique. Pour obtenir l'exemple cherché, il suffit donc de construire un compact $X$ tel que $E(X)$ contienne un ouvert n'ayant pas le type d'homotopie d'un CW-complexe. Il est beaucoup plus facile d'étudier la topologie libre sur $E(X)$, ce qui revient à étudier globalement la collection des espaces métrisables $(E(X), \tau)$ avec $\tau \in \mathcal{T}(X)$, que d'étudier individuellement chacun des espaces $(E(X), \tau)$.

Nous commencerons par donner une description détaillée de l'espace $E(X)$ et en prouver les quelques propriétés élémentaires dont nous avons besoin.

Nous noterons $I$ l'intervalle $[0,1]$. Une distance sur un espace métrisable $X$ sera dite admissible si elle définit la topologie de $X$ (nous aurons aussi à considérer des distances sur des espaces non métrisables, et préciserons systématiquement si nos distances sont admissibles ou seulement continues). Si $d$ est un distance sur un ensemble $X$ et $A, B$ des sous-ensembles de $X$, nous poserons $d(A, B)=\inf _{a \in A, b \in B} d(a, b)$; si $A=\{a\}$ est réduit à un point, nous écrirons $d(a, B)$ au lieu de $d(\{a\}, B)$.

2. L'espace vectoriel topologique libre sur un compact métrisable. Rappelons qu'un espace topologique $Y$ est dit limite inductive d'une suite croissante de fermés $\left\{Y_{n}\right\}_{n=1}^{\infty}$, noté $Y=\underline{\lim } Y_{n}$, si $Y=\bigcup_{n=1}^{\infty} Y_{n}$ et si un sous-ensemble $F$ de $Y$ est fermé dans $Y$ si, et seulement si, $F \cap Y_{n}$ est fermé pour tout $n$. Il est connu que si les $Y_{n}$ sont parfaitement normaux, il en est de même de $Y$. En outre, si $Y=\underline{\lim } Y_{n}$ et $Z=\underline{\lim } Z_{n}$ où les fermés $Y_{n}$ et $Z_{n}$ sont compacts, alors $Y \times Z=\underline{\lim } Y_{n} \times Z_{n}$. Il est aussi connu, et facile de vérifier, que si $Y=\underline{\lim } Y_{n}$, où les $Y_{n}$ sont métrisables, et si $T$ est un rétracte absolu de voisinage, alors toute fonction continue d'un fermé $A$ de $Y$ dans $T$ peut se prolonger à un voisinage de $A$ dans $Y$.

Soit $X$ un espace métrique compact, et soit $E(X)$ l'espace vectoriel réel ayant $X$ pour base. Pour $n \geq 1$, le point générique de $X^{n} \times \mathbb{R}^{n}$ sera noté $(x, \lambda)$, où $x=\left(x_{1}, \ldots, x_{n}\right) \in X^{n}$ et $\lambda=\left(\lambda_{1}, \ldots, \lambda_{n}\right) \in \mathbb{R}^{n}$. Définissons une fonction $\alpha_{n}$ de $X^{n} \times \mathbb{R}^{n}$ dans $E(X)$ par $\alpha_{n}(x, \lambda)=\sum_{i=1}^{n} \lambda_{i} x_{i}$. Pour $t \geq 0$, soient $D_{n}(X, t)=\left\{(x, \lambda) \in X^{n} \times \mathbb{R}^{n}: \sum_{i=1}^{n}\left|\lambda_{i}\right| \leq t\right\}$ et $E_{n}(X, t)=$ $\alpha_{n}\left(D_{n}(X, t)\right)$. Posons $D_{n}(X)=D_{n}(X, n)$ et $E_{n}(X)=E_{n}(X, n)$. Nous munissons chaque ensemble $E_{n}(X)$ de la topologie quotient de $D_{n}(X)$ par $\alpha_{n}$, ce qui en fait un espace compact métrisable. Nous définissons une topologie sur $E(X)$ en convenant qu'un sous-ensemble $A$ de $E(X)$ est ouvert si, est seulement si, $A \cap E_{n}(X)$ est ouvert dans $E_{n}(X)$ pour tout $n \geq 1$. 
$E(X)$ devient ainsi la limite inductive de la suite croissante de compacts $\left\{E_{n}(X)\right\}$.

Les opérations algébriques sont continues sur $E(X)$. En effet, pour tout $n \geq 1$, nous avons un diagramme commutatif

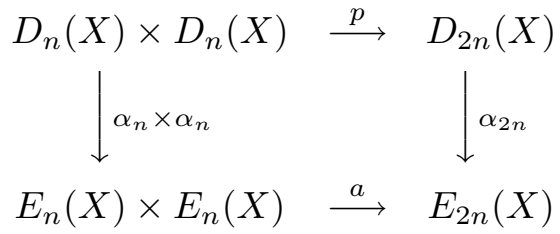

où $a$ est la restriction de l'addition et $p$ est définie par

$$
\begin{aligned}
p\left(\left(x_{1}, \ldots, x_{n}, \lambda_{1}, \ldots, \lambda_{n}\right),\left(x_{n+1}, \ldots, x_{2 n}, \lambda_{n+1}, \ldots, \lambda_{2 n}\right)\right) & \\
& =\left(x_{1}, \ldots, x_{2 n}, \lambda_{1}, \ldots, \lambda_{2 n}\right) .
\end{aligned}
$$

Comme $p, \alpha_{2 n}$ et $\alpha_{n} \times \alpha_{n}$ sont continues et $\alpha_{n} \times \alpha_{n}$ est une application quotient, $a$ est continue. Puisque les $E_{n}(X)$ sont compacts, $E(X) \times E(X)=$ $\varliminf_{n} E_{n}(X) \times E_{n}(X)$, d'où la continuité de l'addition. La continuité de la multiplication scalaire se prouve de même en utilisant le fait que $E(X) \times \mathbb{R}=$ $\varliminf_{n} E_{n}(X) \times[-n, n]$ et le diagramme commutatif

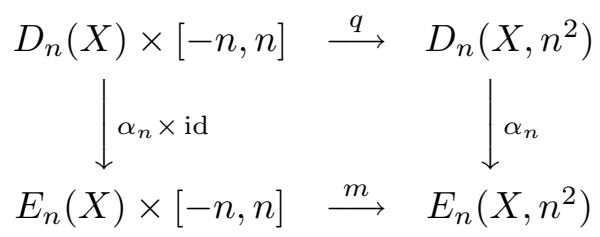

où $q\left(\left(x_{1}, \ldots, x_{n}, \lambda_{1}, \ldots, \lambda_{n}\right), t\right)=\left(x_{1}, \ldots, x_{n}, t \lambda_{1}, \ldots, t \lambda_{n}\right)$ et $m$ est la multiplication.

Si $f$ est une fonction continue de $X$ dans un espace vectoriel topologique $G$, alors l'unique application linéaire $F$ de $E(X)$ dans $G$ qui prolonge $f$ est continue puisque, pour tout $n$, l'application $F \circ \alpha_{n}$ est visiblement continue. Cela justifie d'appeler $E(X)$ l'espace vectoriel topologique libre sur $X$.

Nous noterons $\mathcal{T}(X)$ l'ensemble des topologies d'espace métrique linéaire sur $E(X)$ qui sont moins fines que la topologie libre. Le lemme 2.1 ci-dessous montrera en particulier que la topologie libre est la borne supérieure des éléments de $\mathcal{T}(X)$. Si $\left\{\tau_{i}\right\}_{i=1}^{\infty}$ est une suite d'éléments de $\mathcal{T}(X)$, alors la borne supérieure $\tau$ des topologies $\tau_{i}$ appartient à $\mathcal{T}(X)$. En effet, c'est évidemment une topologie d'espace vectoriel séparé moins fine que la topologie libre, et si, pour $i \geq 1,\left\{V_{i}^{j}\right\}_{j=1}^{\infty}$ est une base dénombrable de $\tau_{i}$-voisinages de 0 , alors les ensembles $V_{i_{1}}^{j_{1}} \cap \ldots \cap V_{i_{k}}^{j_{k}}$ forment une base dénombrable de $\tau$-voisinages de 0 , donc $\tau$ est métrisable.

Si $U$ est un sous-ensemble de $E(X)$, nous noterons encore $U$ l'espace obtenu en munissant $U$ de la topologie induite par la topologie libre. Si $\tau$ 
est une autre topologie sur $E(X)$, nous noterons $(U, \tau)$ l'espace obtenu en munissant $U$ de la topologie induite par $\tau$. D'une façon générale, toute notion topologique non explicitement associée à une topologie $\tau$ sera supposée concerner la topologie libre.

2.1. Lemme. Si $U$ est un ouvert de $E(X)$, il existe $\tau \in \mathcal{T}(X)$ telle que $U$ soit $\tau$-ouvert.

Démonstration. Commençons par montrer que si $V$ est un voisinage de 0 dans $E(X)$, il existe $\tau \in \mathcal{T}(X)$ telle que $V$ soit un $\tau$-voisinage de 0 . Puisque $E(X)$ est limite inductive d'une suite de compacts métrisables, il est parfaitement normal, donc nous pouvons trouver une fonction continue $\delta: E(X) \rightarrow I$ telle que $\delta^{-1}(0)=\{0\}$. Nous pouvons trouver par récurrence une suite $\left\{V_{n}\right\}_{n=1}^{\infty}$ de voisinages de 0 dans $E(X)$ vérifiant

$$
\begin{gathered}
V_{1} \subset V, \\
a V_{n} \subset V_{n} \quad \text { pour tout } a \in[-1,1], \\
V_{n+1}+V_{n+1} \subset V_{n}, \\
V_{n} \subset \delta^{-1}([0,1 / n]) .
\end{gathered}
$$

La condition (4) garantit que $\bigcap_{n=1}^{\infty} V_{n}=\{0\}$, et le corollaire 6.8 de [10] garantit l'existence d'une topologie $\tau$ d'espace métrique linéaire sur $E(X)$ telle que $\left\{V_{n}\right\}$ soit une base de $\tau$-voisinages de 0 . Nécessairement, $\tau$ appartient à $\mathcal{T}(X)$, et $(1)$ garantit que $V$ est un $\tau$-voisinage de 0 .

Pour tout $x \in U$, nous pouvons trouver un voisinage $V_{x}$ de 0 tel que $x+$ $V_{x} \subset U$. D'après ce qui précède, il existe $\tau_{x} \in \mathcal{T}(X)$ telle que $V_{x}$ soit un $\tau_{x^{-}}$ voisinage de 0 . Soit $B_{x}$ le $\tau_{x}$-intérieur de $V_{x}$; les $B_{x}$ sont ouverts dans $E(X)$ et $U=\bigcup_{x \in U}\left(x+B_{x}\right)$. Etant réunion d'une suite de compacts métrisables, $E(X)$ est héréditairement de Lindelöf, donc nous pouvons trouver une suite $\left\{x_{i}\right\}_{i=1}^{\infty}$ de points de $U$ telle que $U=\bigcup_{i=1}^{\infty}\left(x_{i}+B_{x_{i}}\right)$. La borne supérieure $\tau$ de la suite $\left\{\tau_{x_{i}}\right\}_{i=1}^{\infty}$ appartient encore à $\mathcal{T}(X)$ et chacun des ensembles $x_{i}+B_{x_{i}}$ est $\tau$-ouvert, ce qui implique que $U$ est $\tau$-ouvert.

2.2. Lemme. Soient $A$ un sous-espace de $E(X), Y$ un espace métrique et $\varphi: A \rightarrow Y$ une fonction continue. Alors, il existe $\tau \in \mathcal{T}(X)$ telle que $\varphi:(A, \tau) \rightarrow Y$ soit continue.

Démonstration. Pour tout entier $p \geq 1$ et tout point $x$ de $A$, nous pouvons trouver un voisinage ouvert $V_{p}(x)$ de $x$ dans $E(X)$ tel que le diamètre de $\varphi\left(V_{p}(x) \cap A\right)$ soit inférieur à $1 / p$. Le lemme 2.1 nous permet de trouver $\tau_{p}(x) \in \mathcal{T}(X)$ telle que $V_{p}(x)$ soit $\tau_{p}(x)$-ouvert. Puisque $E(X)$ est héréditairement de Lindelöf, nous pouvons trouver une suite $\left\{x_{i}\right\}_{i=1}^{\infty}$ de points de $A$ telle que, pour tout $p, A \subset \bigcup_{i=1}^{\infty} V_{p}\left(x_{i}\right)$. Soit $\tau$ la borne supérieure des topologies $\tau_{p}\left(x_{i}\right)(i, p \geq 1)$. Alors $\tau \in \mathcal{T}(X)$ et, pour tout $p \geq 1$, la suite $\left\{V_{p}\left(x_{i}\right)\right\}_{i=1}^{\infty}$ est un recouvrement $\tau$-ouvert de $A$. Comme le 
diamètre de chaque ensemble $\varphi\left(V_{p}(x) \cap A\right)$ est inférieur à $1 / p$, cela entraîne la continuité de $\varphi:(A, \tau) \rightarrow Y$.

2.3. Lemme. Si $X$ est de dimension finie, alors $E(X)$ est réunion dénombrable de compacts de dimension finie.

Dém on stration. Il suffit de vérifier que, pour $n \geq 1$ et $t>0, E_{n}(X, t)$ est de dimension finie. Le cas $n=1$ étant évident, cela peut se faire par récurrence en remarquant que tout point de $E_{n}(X, t) \backslash E_{n-1}(X, t)$ a, dans $E_{n}(X, t)$, un voisinage homéomorphe à un sous-ensemble de $X^{n} \times \mathbb{R}^{n}$.

3. Propriétés d'extension des $(E(X), \tau)$. Pour prouver le théorème 1.1 et son addendum, nous cherchons un compact $X$ pour lequel il existe $\tau \in \mathcal{T}(X)$ telle que $(E(X), \tau)$ ne soit pas un rétracte absolu. Cependant, nous avons besoin de connaître deux cas particuliers où il ne peut exister de telle topologie $\tau$.

3.1. Lemme. Si $X$ est de dimension finie, alors $(E(X), \tau)$ est un rétracte absolu quelle que soit $\tau \in \mathcal{T}(X)$.

Si $K$ est un compact de $E(X)$, alors $(K, \tau)$ est homéomorphe à $K$, donc, compte-tenu du lemme 2.3, cela est un cas particulier d'un théorème de Haver [8].

Le lemme suivant sera utilisé pour prouver l'addendum 1.2.

3.2. Lemme. Si $X$ est un rétracte absolu de voisinage, alors $(E(X), \tau)$ est un rétracte absolu quelle que soit $\tau \in \mathcal{T}(X)$.

Pour prouver ce lemme, nous avons besoin d'un résultat préliminaire.

3.3. Lemme. Si un espace métrique linéaire $E$ est réunion d'une suite $\left\{E_{n}\right\}_{n=1}^{\infty}$ de fermés qui sont des rétractes absolus de voisinage, alors $E$ est un rétracte absolu.

Démonstration. Fixons une distance admissible $d$ sur $E$. Il suffit de prouver que $E$ est un rétracte absolu de voisinage. Soient $A$ un fermé d'un espace métrique $Z$ et $f$ une fonction continue de $A$ dans $E$. Pour prouver que $f$ se prolonge à un voisinage de $A$, il suffit, d'après le lemme 1 de [4], de montrer que, pour tout $\varepsilon>0$, il existe une fonction continue $f_{\varepsilon}: A \rightarrow E$ vérifiant

$$
d\left(f(x), f_{\varepsilon}(x)\right)<\varepsilon \quad \forall x \in A,
$$

$f_{\varepsilon}$ peut se prolonger à un voisinage de $A$.

Pour $n \geq 1$, soit $A_{n}=f^{-1}\left(E_{n}\right)$; alors $\left\{A_{n}\right\}_{n=1}^{\infty}$ est une suite de fermés dont la réunion est $A$. Nous allons construire par récurrence des voisinages 
ouverts $V_{n}$ et $W_{n}$ de $A_{1} \cup \ldots \cup A_{n}$ dans $Z$ et des fonctions continues $h_{n}$ : $V_{n} \rightarrow E$ de façon que, pour tout $n \geq 1$,

$$
\begin{gathered}
\bar{W}_{n} \subset V_{n}, \\
W_{n} \subset W_{n+1}, \\
d\left(f(x), t f(x)+(1-t) h_{n}(x)\right)<\varepsilon \quad \text { pour } x \in A \cap V_{n} \text { et } t \in I, \\
h_{n+1}\left|\bar{W}_{n}=h_{n}\right| \bar{W}_{n} .
\end{gathered}
$$

Puisque les $E_{n}$ sont des rétractes absolus de voisinage, nous pouvons trouver, pour tout $n \geq 1$, un voisinage ouvert $U_{n}$ de $A_{n}$ dans $Z$ et une fonction continue $g_{n}: U_{n} \rightarrow E_{n}$ prolongeant $f \mid A_{n}$. Quitte à remplacer $U_{n}$ par un voisinage plus petit, nous pouvons supposer que

$$
d\left(f(x), t f(x)+(1-t) g_{n}(x)\right)<\varepsilon \quad \text { pour } x \in A \cap U_{n} \text { et } t \in I .
$$

Posons $V_{1}=U_{1}$ et $h_{1}=g_{1}$, puis prenons un voisinage ouvert $W_{1}$ de $A_{1}$ tel que $\bar{W}_{1} \subset V_{1}$. Soit maintenant $n \geq 1$, et supposons $V_{n}, W_{n}$ et $h_{n}$ construits. D'après (3), les fermés $\bar{W}_{n}$ et $A_{n+1} \backslash V_{n}$ sont disjoints, donc nous pouvons trouver des ouverts $O$ et $P$ dont les fermetures sont disjointes et vérifiant

$$
\begin{gathered}
\bar{W}_{n} \subset O \subset \bar{O} \subset V_{n}, \\
A_{n+1} \backslash V_{n} \subset P \subset \bar{P} \subset U_{n+1} .
\end{gathered}
$$

Soit $\alpha: Z \rightarrow I$ une fonction continue telle que $\alpha^{-1}(0)=\bar{O}$ et $\alpha^{-1}(1)=\bar{P}$. L'ensemble $A_{n+1} \backslash(O \cup P)$ est fermé et contenu dans $V_{n} \cap U_{n+1}$. Nous prendrons $V_{n+1}$ de la forme $V_{n+1}=O \cup P \cup H$ où $H$ est un voisinage de $A_{n+1} \backslash(O \cup P)$ contenu dans $V_{n} \cap U_{n+1}$, et nous définirons $h_{n+1}$ par

$$
h_{n+1}(x)= \begin{cases}h_{n}(x) & \text { si } x \in O, \\ (1-\alpha(x)) h_{n}(x)+\alpha(x) g_{n+1}(x) & \text { si } x \in H, \\ g_{n+1}(x) & \text { si } x \in P .\end{cases}
$$

La condition (6) résultera alors de (8). Reste à choisir $H$ de façon que pour tout $x \in V_{n+1} \cap A$, on ait

$$
d\left(f(x), t f(x)+(1-t) h_{n+1}(x)\right)<\varepsilon \quad \text { pour tout } t \in I .
$$

Remarquons d'abord que les conditions (5) et (8) (resp. (7) et (9)) garantissent que (10) est vérifiée par tout point $x$ de $O \cap A$ (resp. $P \cap A$ ). En outre, si $x \in A_{n+1} \backslash(O \cup P)$, alors $g_{n+1}(x)=f(x)$ et $h_{n+1}(x)=$ $(1-\alpha(x)) h_{n}(x)+\alpha(x) f(x)$ appartient au segment d'extrémités $f(x)$ et $h_{n}(x)$. La condition (5) garantit alors que (10) est vérifiée par tout point $x$ de $A_{n+1} \backslash(O \cup P)$. Par continuité, elle est donc vérifiée par tout point de $H \cap A$, pourvu que $H$ soit un voisinage assez petit de $A_{n+1} \backslash(O \cup P)$. L'ouvert $V_{n+1}$ ainsi construit contient $\bar{W}_{n} \cup A_{n+1} \supset A_{1} \cup \ldots \cup A_{n+1}$ et, pour achever la construction, il suffit de choisir un ouvert $W_{n+1}$ tel que $\bar{W}_{n} \cup A_{n+1} \subset W_{n+1} \subset \bar{W}_{n+1} \subset V_{n+1}$. 
Alors, $W=\bigcup_{n=1}^{\infty} W_{n}$ est un voisinage de $A$ dans $Z$ et la fonction $h$ : $W \rightarrow E$ définie par $h\left|W_{n}=h_{n}\right| W_{n}$ pour tout $n$ est continue et, d'après (5), vérifie $d(f(x), h(x))<\varepsilon$ pour tout $x \in A$, donc $f_{\varepsilon}=h \mid A$ vérifie les conditions (1) et (2), d'où le lemme.

Démonstration du lemme 3.2. Puisque $E(X)=\bigcup_{n=1}^{\infty} E_{n}(X)$, il suffit, d'après le lemme 3.3 , de montrer que chaque $E_{n}(X)$ est un rétracte absolu. $E_{n}$ peut être regardé comme un foncteur de la catégorie des espaces compacts dans elle-même, le morphisme associé à une fonction continue $f$ de $X$ dans $Y$ étant la restriction à $E_{n}(X)$ de l'application linéaire continue de $E(X)$ dans $E(Y)$ qui prolonge $f$ (on convient que $E_{n}(\emptyset)=\{0\}$ ). Ce foncteur a les propriétés suivantes, dont les vérifications élémentaires sont laissées au lecteur :

(a) $E_{n}$ est continu, i.e. commute aux limites projectives,

(b) si $f: X \rightarrow Y$ est injective, alors $E_{n}(f): E_{n}(X) \rightarrow E_{n}(Y)$ est injective,

(c) si $\left\{X_{\alpha}\right\}_{\alpha \in A}$ est une famille de fermés d'un espace $X$, alors $E_{n}\left(\bigcap_{\alpha \in A} X_{\alpha}\right)=\bigcap_{\alpha \in A} E_{n}\left(X_{\alpha}\right)$,

(d) pour tout $y \in E_{n}(X)$, il existe un sous-espace $X^{\prime}$ de $X$ de cardinal $\leq n$ tel que $y \in E_{n}\left(X^{\prime}\right)$,

(e) si $X$ est fini, alors $E_{n}(X)$ est un polyèdre.

D'après un résultat de $\mathrm{V}$. N. Basmanov ([1], théorème 2), ces propriétés entraînent que $E_{n}(X)$ est un rétracte absolu de voisinage chaque fois que $X$ en est un (la démonstration du théorème 2 de [1] s'adapte au cas où $F(\emptyset)$ est un rétracte absolu de voisinage de dimension finie au lieu d'être supposé vide).

4. Démonstration du théorème 1.1. Dans cette section, nous fixons un compact $X$, un polyèdre fini $Y$ et une fonction $f$ de $Y$ dans $X$ vérifiant

(i) $X$ est de dimension infinie,

(ii) $f$ est une surjection continue ouverte,

(iii) pour tout $x \in X, f^{-1}(x)$ est de forme triviale.

Un tel triplet $(X, Y, f)$ peut s'obtenir comme suit. D'après A. N. Dranishnikov [5], il existe un compact $Z$ de dimension trois et une décomposition semi-continue supérieurement $\mathcal{D}$ de $Z$ telle que chaque élément de $\mathcal{D}$ soit de forme triviale et que le quotient $Z / \mathcal{D}$ soit de dimension infinie. Plongeons $Z$ dans $Y=S^{7}$, et soit $\mathcal{D}^{\prime}$ la décomposition semi-continue supérieurement de $S^{7}$ dont les éléments sont ceux de $\mathcal{D}$ et les points de $S^{7} \backslash Z$. Alors, $X=S^{7} / \mathcal{D}^{\prime}$ est de dimension infinie et un théorème de J. J. Walsh [13] garantit l'existence d'une fonction $f$ de $S^{7}$ sur $X$ vérifiant (ii) et (iii). 
Fixons un entier $m>\operatorname{dim} Y$. Puisque $X$ est de dimension infinie, nous pouvons trouver un fermé $A$ de $X$ et une fonction continue $g$ de $A$ dans la sphère $S^{m}$ n'admettant aucun prolongement à $X$ ([7], théorème 3.2.10). Puisque $S^{m}$ est un rétracte absolu de voisinage, nous pouvons trouver un voisinage ouvert $W$ de $A$ dans $E(X)$ et une fonction continue $\bar{g}: \bar{W} \rightarrow S^{m}$ prolongeant $g$; il sera commode de supposer que $\bar{W}$ ne contient pas 0 . Soit $F: E(Y) \rightarrow E(X)$ l'application linéaire continue prolongeant $f$. Le lemme suivant, qui est le coeur de la démonstration, sera prouvé plus loin.

4.1. LEMME. Il existe un voisinage ouvert $U$ de $X \cup \bar{W}$ dans $E(X)$ et une fonction continue $h: F^{-1}(U) \rightarrow S^{m}$ telle que $h \mid F^{-1}(\bar{W})=\bar{g} \circ\left(F \mid F^{-1}(\bar{W})\right)$.

Les lemmes 2.1 et 2.2 garantissent l'existence de topologies $\tau \in \mathcal{T}(X)$ telles que $U$ et $W$ soient $\tau$-ouverts et que $\bar{g}:(\bar{W}, \tau) \rightarrow S^{m}$ soit continue. Le théorème 1.1 résulte donc du suivant.

4.2. ThÉORÈme. Soit $\tau \in \mathcal{T}(X)$ telle que $U$ et $W$ soient $\tau$-ouverts et que $\bar{g}:(\bar{W}, \tau) \rightarrow S^{m}$ soit continue. Alors, $(E(X), \tau)$ n'est pas un rétracte absolu.

Démonstration. Supposons au contraire que $(E(X), \tau)$ soit un rétracte absolu. Le lemme 2.2 permet de trouver $\tau^{\prime} \in \mathcal{T}(Y)$ telle que $F$ : $\left(E(Y), \tau^{\prime}\right) \rightarrow(E(X), \tau)$ et $h:\left(F^{-1}(U), \tau^{\prime}\right) \rightarrow S^{m}$ soient continues.

Affirmation. Pour tout sous-ensemble $\tau$-ouvert $V$ de $E(X)$, $F \mid F^{-1}(V):\left(F^{-1}(V), \tau^{\prime}\right) \rightarrow(V, \tau)$ est une équivalence homotopique.

Pr e u ve. Puisque $\left(E(Y), \tau^{\prime}\right)$ est un rétracte absolu d'après le lemme 3.1, l'ouvert $\left(F^{-1}(V), \tau^{\prime}\right)$ a le type d'homotopie d'un CW-complexe. Puisque $(E(X), \tau)$ est supposé être un rétracte absolu, $(V, \tau)$ a aussi le type d'homotopie d'un CW-complexe. Il suffit donc de vérifier que $F \mid F^{-1}(V)$ est une équivalence homotopique faible, ce qui résulte immédiatement d'un théorème de G. Kozlowski [12] (noter que, pour tout $\tau$-voisinage étoilé $N$ d'un point quelconque de $E(X)$, les ensembles $(N, \tau)$ et $\left(F^{-1}(N), \tau^{\prime}\right)$ sont contractiles, ce qui entraîne que l'hypothèse du théorème 1 de [12] est vérifiée pour tout $n)$.

Soient $F_{1}:\left(F^{-1}(U), \tau^{\prime}\right) \rightarrow(U, \tau)$ et $F_{2}:\left(F^{-1}(W), \tau^{\prime}\right) \rightarrow(W, \tau)$ les restrictions de $F$; ce sont donc des équivalences homotopiques. Puisque $F_{1}$ est une équivalence homotopique, il existe une fonction continue $k:(U, \tau) \rightarrow$ $S^{m}$ telle que $h \mid\left(F^{-1}(U), \tau^{\prime}\right)$ soit homotope à $k \circ F_{1}$. Puisque $h \mid F^{-1}(W)=$ $(\bar{g} \mid W) \circ F_{2}$ et que $F_{2}$ est une équivalence homotopique, $k \mid W$ et $\bar{g} \mid W$ sont homotopes. En particulier, $g$ est homotope à $k \mid A$ et, comme $k \mid X$ est un prolongement de $k \mid A$ à $X$, le théorème d'extension des homotopies de Borsuk garantit que $g$ se prolonge à $X$, ce qui contredit le choix de $g$. 
Preuve de l'addendum. Plongeons $X$ dans le cube de Hilbert $Q$. Alors, $E(X)$ est un sous-espace vectoriel fermé de $E(Q)$. Si $U^{\prime}$ et $W^{\prime}$ sont des ouverts de $E(Q)$ tels que $U^{\prime} \cap E(X)=U$ et $W^{\prime} \cap E(X)=W$, les lemmes 2.1 et 2.2 permettent de trouver $\tau_{0} \in \mathcal{T}(Q)$ telle que $U^{\prime}, W^{\prime}$ et $E(Q) \backslash E(X)$ soient $\tau_{0}$-ouverts et que $\bar{g}:\left(\bar{W}, \tau_{0}\right) \rightarrow S^{m}$ soit continue. Alors, la topologie $\tau$ induite par $\tau_{0}$ sur $E(X)$ vérifie les hypothèses du théorème 4.2 , et $(E(X), \tau)$ est un sous-espace fermé de $\left(E(Q), \tau_{0}\right)$, qui est un rétracte absolu d'après le lemme 3.2 .

5. Démonstration du lemme 4.1. Pour tout espace topologique $Z$, nous notons $2^{Z}$ l'espace des compacts de $Z$ muni de la topologie de Vietoris. Il est connu que si $f: Y \rightarrow X$ est une surjection continue entre espaces compacts, alors la fonction $\varphi: X \rightarrow 2^{Y}$ définie par $\varphi(x)=f^{-1}(x)$ est continue si, et seulement si, $f$ est ouverte. Nous aurons besoin du résultat suivant, qui est un cas particulier d'un théorème de W. E. Haver [9].

5.1. Lemme. Soient $Z$ un espace métrique qui est réunion dénombrable de compacts de dimension finie et $T$ un rétracte absolu de voisinage. Soit $\varphi: Z \rightarrow 2^{T}$ une fonction continue telle que, pour tout $z \in Z, \varphi(z)$ soit de forme triviale. Alors, si d est une distance admissible sur $T$ et $\varepsilon: Z \rightarrow] 0,1]$ une fonction continue, il existe une fonction continue $\chi: Z \rightarrow T$ telle que $d(\chi(z), \varphi(z))<\varepsilon(z)$ pour tout $z \in Z$.

Posons $G_{0}(X)=\{0\}$ et $G_{n}(X)=\alpha_{n}\left(X^{n} \times \mathbb{R}^{n}\right)$ pour $n>1$. Pour $n \geq 1$, soit $H_{n}(X)=G_{n}(X) \backslash G_{n-1}(X)$. Les ensembles $G_{n}(Y), n \geq 0$, et $H_{n}(Y)$, $n \geq 1$, sont définis de façon analogue. Il est facile de voir que, pour tout $n \geq 0, G_{n}(X)$ est fermé dans $E(X)$. Tout point $z$ de $H_{n}(X)$ peut se mettre sous la forme $z=\sum_{i=1}^{n} \lambda_{i} x_{i}$ où $x_{1}, \ldots, x_{n}$ sont des points distincts de $X$ et les $\lambda_{i}$ des réels $\neq 0$; cette représentation est unique à l'ordre près. Nous avons besoin d'une remarque élémentaire.

5.2. Lemme. Soient $1 \leq n \leq p$ des entiers. Si $z=\sum_{i=1}^{n} \lambda_{i} x_{i}$ est un point de $H_{n}(X) \cap E_{p}(X)$, alors $\sum_{i=1}^{n}\left|\lambda_{i}\right| \leq p$.

Démonstration. Puisque $z$ appartient à $E_{p}(X)$, il peut s'écrire $z=$ $\sum_{j=1}^{p} \mu_{j} y_{j}$ où $\sum_{j=1}^{p}\left|\mu_{j}\right| \leq p$. Pour $i=1, \ldots, n$, soit $N_{i}$ l'ensemble des indices $j$ tels que $y_{j}=x_{i}$. Alors, $\sum_{j \in N_{i}} \mu_{j}=\lambda_{i}$, donc $\left|\lambda_{i}\right| \leq \sum_{j \in N_{i}}\left|\mu_{j}\right|$; le lemme en résulte.

5.3. Lemme. Si $0<t<t^{\prime}$, alors $E_{n}\left(Y, t^{\prime}\right) \cap H_{n}(Y)$ est un voisinage de $E_{n}(Y, t) \cap H_{n}(Y)$ dans $H_{n}(Y)$.

Démonstration. Soit $E_{n}^{\prime}\left(t^{\prime}\right)$ l'ensemble des points $y=\sum_{i=1}^{n} \lambda_{i} y_{i}$ de $H_{n}(Y)$ tels que $\sum_{i=1}^{n}\left|\lambda_{i}\right|<t^{\prime}$. Alors $E_{n}(Y, t) \cap H_{n}(Y) \subset E_{n}^{\prime}\left(t^{\prime}\right) \subset$ $E_{n}\left(Y, t^{\prime}\right) \cap H_{n}(Y)$, et il suffit de vérifier que $E_{n}^{\prime}\left(t^{\prime}\right)$ est ouvert dans $G_{n}(Y)$. Comme $G_{n}(Y)$ est fermé, $G_{n}(Y)=\varliminf_{q} G_{n}(Y) \cap E_{q}(Y)$ et, comme $E_{q}(Y)$ 
est un compact métrisable, il suffit de vérifier qu'aucune suite de points de $E_{q}(Y) \cap\left(G_{n}(Y) \backslash E_{n}^{\prime}\left(t^{\prime}\right)\right)$ ne peut converger vers un point de $E_{n}^{\prime}\left(t^{\prime}\right)$, ce qui est facile.

5.4. Lemme. Pour tout $n \geq 1, H_{n}(Y)$ est un rétracte absolu de voisinage.

Démonstration. Il est facile de déduire du lemme 5.3 que la restriction de $\alpha_{n}$ à $Y^{n} \times \mathbb{R}^{n} \backslash \alpha_{n}^{-1}\left(G_{n-1}(Y)\right)$ est un revêtement à $n$ ! feuillets. Cet ensemble étant ouvert dans $Y^{n} \times \mathbb{R}^{n}$ et $Y$ étant un polyèdre, le lemme en résulte.

Soit $n \geq 1$. Si $z=\sum_{i=1}^{n} \lambda_{i} x_{i}$ est un point de $H_{n}(X)$, l'ensemble

$$
\begin{aligned}
\varphi_{n}(z) & =\lambda_{1} f^{-1}\left(x_{1}\right)+\ldots+\lambda_{n} f^{-1}\left(x_{n}\right) \\
& =\left\{\lambda_{1} a_{1}+\ldots+\lambda_{n} a_{n}: a_{i} \in f^{-1}\left(x_{i}\right) \text { pour } i=1, \ldots, n\right\}
\end{aligned}
$$

est un compact contenu dans $H_{n}(Y)$.

5.5. Lemme. La fonction $\varphi_{n}: H_{n}(X) \rightarrow 2^{H_{n}(Y)}$ est continue et, pour tout $z \in H_{n}(X), \varphi_{n}(z)$ est de forme triviale.

Démonstration. Comme la restriction $\alpha_{n}^{\prime}$ de $\alpha_{n}$ à $X^{n} \times \mathbb{R}^{n} \backslash$ $\alpha_{n}^{-1}\left(G_{n-1}(X)\right)$ est un revêtement de cet espace sur $H_{n}(X)$, il suffit, pour prouver la continuité de $\varphi_{n}$, de vérifier que $\varphi_{n} \circ \alpha_{n}^{\prime}$ est continue, ce qui résulte immédiatement de la continuité de la fonction $x \rightsquigarrow f^{-1}(x)$ de $X$ dans $2^{Y}$. Pour $z=\sum_{i=1}^{n} \lambda_{i} x_{i} \in H_{n}(X)$, l'application $\left(a_{1}, \ldots, a_{n}\right) \rightsquigarrow \lambda_{1} a_{1}+\ldots+\lambda_{n} a_{n}$ est un homéomorphisme du produit $f^{-1}\left(x_{1}\right) \times \ldots \times f^{-1}\left(x_{n}\right)$ sur $\varphi_{n}(z)$; comme les compacts $f^{-1}\left(x_{1}\right), \ldots, f^{-1}\left(x_{n}\right)$ sont de forme triviale, il en est de même de leur produit.

Choisissons une distance continue $d_{Y}$ sur $E(Y)$ vérifiant

(1) Les ensembles $F^{-1}(\bar{W})$ et $F^{-1}\left(G_{n}(X)\right), n \geq 1$, sont $d_{Y}$-fermés.

Pour construire $d_{Y}$, il suffit d'observer que $F^{-1}(\bar{W})$ et $F^{-1}\left(G_{n}(X)\right)$ sont fermés et, $d$ étant une distance continue arbitraire sur $E(Y)$, de poser $d_{Y}(x, y)=d(x, y)+\sum_{n=0}^{\infty} 2^{-n}\left|\gamma_{n}(x)-\gamma_{n}(y)\right|$ où les $\gamma_{n}$ sont des fonctions continues de $E(Y)$ dans $I$ telles que $\gamma_{0}^{-1}(0)=F^{-1}(\bar{W})$ et $\gamma_{n}^{-1}(0)=$ $F^{-1}\left(G_{n}(X)\right)$ pour $n \geq 1$.

Pour tout $n \geq 1$ et tout sous-ensemble $B$ de $G_{n}(x)$, nous noterons $\operatorname{Int}_{n} B$ l'intérieur de $B$ relativement à $G_{n}(X)$. Nous allons construire, pour $n \geq 1$, des voisinages fermés $V_{n}, V_{n}^{\prime}$ de $X \cup\left(\bar{W} \cap G_{n}(X)\right)$ dans $G_{n}(X)$ et une fonction continue $k_{n}: F^{-1}\left(V_{n}^{\prime} \cup \bar{W}\right) \rightarrow S^{m}$ vérifiant, pour tout $n$,

$$
\begin{gathered}
V_{n} \subset \operatorname{Int}_{n} V_{n}^{\prime}, \\
V_{n+1} \cap G_{n}(X)=V_{n} \quad \text { et } \quad \operatorname{Int}_{n+1} V_{n+1} \cap G_{n}(X)=\operatorname{Int}_{n} V_{n}, \\
k_{n} \mid F^{-1}(\bar{W})=\bar{g} \circ\left(F \mid F^{-1}(\bar{W})\right), \\
k_{n+1}\left|F^{-1}\left(V_{n} \cup \bar{W}\right)=k_{n}\right| F^{-1}\left(V_{n} \cup \bar{W}\right) .
\end{gathered}
$$


Supposons ces objets construits. Puisque $G_{n}(X)$ est un fermé contenant $E_{n}(X)$, nous avons $E(X)=\underline{\varliminf} G_{n}(X)$, donc $(3)$ garantit que $V=\bigcup_{n=1}^{\infty} V_{n}$ est un fermé d'intérieur $U=\bigcup_{n=1}^{\infty} \operatorname{Int}_{n} V_{n}$; cet ouvert contient $X \cup \bar{W}$. Puisque $F^{-1}\left(G_{n}(X)\right)$ contient $G_{n}(Y) \supset E_{n}(Y)$, nous avons $F^{-1}(V)=$ $\varliminf F^{-1}\left(V_{n} \cup \bar{W}\right)$; d'après (5), nous pouvons définir une fonction continue $k: F^{-1}(V) \rightarrow S^{m}$ par $k\left|F^{-1}\left(V_{n} \cup \bar{W}\right)=k_{n}\right| F^{-1}\left(V_{n} \cup \bar{W}\right)$ pour tout $n$. Alors, (4) garantit que $h=k \mid F^{-1}(U)$ vérifie la conclusion du lemme 4.1.

La construction des $V_{n}, V_{n}^{\prime}$ et $k_{n}$ se fera par récurrence, la première étape de cette récurrence étant un peu différente des autres.

Construction de $V_{1}, V_{1}^{\prime}$ et $k_{1}$. Puisque $\operatorname{dim} Y<m$, la fonction $\bar{g} \circ$ $\left(F \mid F^{-1}(\bar{W})\right)$ peut se prolonger en une fonction continue $k_{0}$ de $Y \cup F^{-1}(\bar{W})$ dans $S^{m}$. Comme $S^{m}$ est un rétracte absolu de voisinage, nous pouvons trouver un voisinage ouvert $P_{0}$ de $Y \cup F^{-1}(\bar{W})$ dans $E(Y)$ et un prolongement continu $\bar{k}_{0}$ de $k_{0}$ à $P_{0}$. Posons $M_{1}=\left\{z \in H_{1}(X): \varphi_{1}(z) \subset P_{0}\right\}$. Puisque $H_{1}(X)$ est ouvert dans $G_{1}(X)$ et $\varphi_{1}$ continue, l'ensemble $M_{1}$ est ouvert dans $G_{1}(X)$. Si $x$ appartient à $X, \varphi_{1}(x)=f^{-1}(x) \subset Y \subset P_{0}$, donc $M_{1}$ contient $X$; puisque nous avons supposé que $\bar{W}$ ne contient pas $0, \bar{W} \cap G_{1}(X)$ est aussi contenu dans $M_{1}$. Nous prendrons pour $V_{1}, V_{1}^{\prime}$ des voisinages fermés de $X \cup\left(\bar{W} \cap G_{1}(X)\right)$ dans $G_{1}(X)$ vérifiant $V_{1} \subset \operatorname{Int}_{1} V_{1}^{\prime} \subset V_{1}^{\prime} \subset M_{1}$. Nous pouvons alors définir une fonction continue $\widetilde{\varphi}_{1}: F^{-1}\left(V_{1}^{\prime}\right) \rightarrow 2^{H_{1}(Y)}$ par $\widetilde{\varphi}_{1}(y)=\varphi_{1}(F(y))$. D'après le lemme $5.4, H_{1}(Y)$ est métrisable; soit $d_{1}$ une distance admissible sur $H_{1}(Y)$. Comme $F^{-1}\left(V_{1}^{\prime}\right)$ et $F^{-1}(\bar{W})$ sont fermés dans $E(Y)$, il résulte du lemme 2.3 que $F^{-1}\left(V_{1}^{\prime} \backslash \bar{W}\right)$ est réunion dénombrable de compacts de dimension finie et, pour tout $\tau \in \mathcal{T}(Y),\left(F^{-1}\left(V_{1}^{\prime} \backslash \bar{W}\right), \tau\right)$ est aussi réunion dénombrable de tels compacts. Le lemme 2.2 nous permet de trouver $\tau \in \mathcal{T}(Y)$ telle que les fonctions $\widetilde{\varphi}_{1}$ et $d_{Y}\left(\cdot, F^{-1}(\bar{W})\right)$ soient $\tau$ continues sur $F^{-1}\left(V_{1}^{\prime} \backslash \bar{W}\right)$ et, en appliquant le lemme 5.1 à $\left(F^{-1}\left(V_{1}^{\prime} \backslash \bar{W}\right), \tau\right)$, nous pouvons trouver une fonction continue $\chi_{1}: F^{-1}\left(V_{1}^{\prime} \backslash \bar{W}\right) \rightarrow H_{1}(Y)$ vérifiant, pour tout $y \in F^{-1}\left(V_{1}^{\prime} \backslash \bar{W}\right)$,

$$
d_{1}\left(\chi_{1}(y), \widetilde{\varphi}_{1}(y)\right)<\min \left(d_{1}\left(\widetilde{\varphi}_{1}(y), H_{1}(Y) \backslash P_{0}\right), d_{Y}\left(y, F^{-1}(\bar{W})\right)\right),
$$

la condition (1) garantissant que le terme de droite de cette inégalité est $>0$. La relation (6) garantit que $\chi_{1}(y)$ appartient à $P_{0}$, ce qui nous permet de définir une fonction $k_{1}$ de $F^{-1}\left(V_{1}^{\prime} \cup \bar{W}\right)$ dans $S^{m}$ par

$$
k_{1}(y)= \begin{cases}\bar{g}(F(y)) & \text { si } y \in F^{-1}(\bar{W}), \\ \bar{k}_{0}\left(\chi_{1}(y)\right) & \text { si } y \in F^{-1}\left(V_{1}^{\prime} \backslash \bar{W}\right) .\end{cases}
$$

La condition (4) étant vérifiée par définition, il reste à établir la continuité de $k_{1}$. Comme $F^{-1}\left(V_{1}^{\prime} \cup \bar{W}\right)$ est fermé, c'est la limite inductive de la suite croissante de compacts métrisables $F^{-1}\left(V_{1}^{\prime} \cup \bar{W}\right) \cap E_{q}(Y)$. Par suite, pour vérifier la continuité de $k_{1}$, il suffit de montrer que si $\{z(p)\}_{p=1}^{\infty}$ est une suite de points de $F^{-1}\left(V_{1}^{\prime} \cup \bar{W}\right)$ convergeant vers un point $z$ de cet ensem- 
ble, alors $\left\{k_{1}(z(p))\right\}$ tend vers $k_{1}(z)$. Puisque $\bar{k}_{0}, \chi_{1}, F$ et $\bar{g}$ sont continue là où elles sont définies, le seul cas nécessitant une vérification est celui où $z \in F^{-1}(\bar{W})$ et où $z(p) \in F^{-1}\left(V_{1}^{\prime} \backslash \bar{W}\right)$ pour tout $p \geq 1$. Alors, $\{F(z(p))\}$ tend vers $F(z)$, qui appartient à $V_{1}^{\prime} \subset H_{1}(X)$ puisque $V_{1}^{\prime}$ est fermé, donc $\left\{\widetilde{\varphi}_{1}(z(p))\right\}$ tend vers $\varphi_{1}(F(z))$. Comme $\left\{d_{Y}\left(z(p), F^{-1}(\bar{W})\right)\right\}$ tend vers zéro, il résulte alors de $(6)$ que l'ensemble $\varphi_{1}(F(z)) \cup\left\{\chi_{1}(z(p)): p \geq 1\right\}$ est un compact de l'espace métrisable $H_{1}(Y)$. Alors, toute sous-suite de la suite $\{z(p)\}$ a une sous-suite $\left\{z\left(p_{k}\right)\right\}_{k=1}^{\infty}$ telle que $\left\{\chi_{1}\left(z\left(p_{k}\right)\right)\right\}$ converge vers un point $a$ de $\varphi_{1}(F(z)) \subset F^{-1}(F(z))$. Mais alors, $\left\{k_{0}\left(\chi_{1}\left(z\left(p_{k}\right)\right)\right)\right\}$ converge vers $\bar{k}_{0}(a)=\bar{g}(F(a))=\bar{g}(F(z))=k_{0}(z)=k_{1}(z)$, ce qui montre que toute sous-suite de la suite $\left\{k_{1}(z(p))\right\}$ a une sous-suite convergeant vers $k_{1}(z)$, donc que $\left\{k_{1}(z(p))\right\}$ tend vers $k_{1}(z)$, d'où la continuité de $k_{1}$.

Soit maintenant $n>1$, et supposons $V_{n-1}, V_{n-1}^{\prime}$ et $k_{n-1}$ construits. Puisque $S^{m}$ est un rétracte absolu de voisinage, nous pouvons trouver un voisinage ouvert $P_{n-1}$ de $F^{-1}\left(V_{n-1}^{\prime} \cup \bar{W}\right)$ dans $E(Y)$ et un prolongement continu $\bar{k}_{n-1}$ de $k_{n-1}$ à $P_{n-1}$. Posons $M_{n}=\left\{z \in H_{n}(X): \varphi_{n}(z) \subset P_{n-1}\right\}$ et $N_{n}=M_{n} \cup \operatorname{Int}_{n-1} V_{n-1}^{\prime}$.

Affirmation 1. $N_{n}$ est ouvert dans $G_{n}(X)$.

Puisque $G_{n}(X)$ est fermé dans $E(X)$, nous avons $G_{n}(X)=\underline{\lim }_{q} G_{n}(X) \cap$ $E_{q}(X)$. Si l'affirmation était fausse, il existerait un $q$ tel que $N_{n} \cap E_{q}(X)$ ne soit pas ouvert dans $G_{n}(X) \cap E_{q}(X)$. Comme $H_{n}(X)$ est ouvert dans $G_{n}(X)$ et $\varphi_{n}$ continue, l'ensemble $M_{n}$ est ouvert dans $G_{n}(X)$. Il devrait donc exister un $x \in \operatorname{Int}_{n-1} V_{n-1}^{\prime}$ tel que $N_{n} \cap E_{q}(X)$ ne soit pas un voisinage de $x$ dans $G_{n}(X) \cap E_{q}(X)$. Comme $E_{q}(X)$ est métrisable, nous pourrions alors trouver une suite $\{z(p)\}_{p=1}^{\infty}$ de points de $E_{q}(X) \cap\left(G_{n}(X) \backslash N_{n}\right)$ convergeant vers $x$. Comme $x$ ne peut pas être limite d'une suite de points de $G_{n-1}(X) \backslash \operatorname{Int}_{n-1} V_{n-1}^{\prime}$, nous pouvons supposer que, pour tout $p, z(p) \in$ $H_{n}(X) \backslash M_{n}$, et il existe donc un point $y(p) \in \varphi_{n}(z(p)) \backslash P_{n-1}$. Comme $z(p)$ appartient à $E_{q}(X)$, le lemme 5.2 garantit que $\varphi_{n}(z(p))$ est contenu dans $E_{q}(Y)$. Comme $E_{q}(Y)$ est un compact métrisable, nous pouvons, quitte à extraire une sous-suite, supposer que $\{y(p)\}$ converge vers un point $y . F$ étant continue, $F(y)=x$. Mais alors, $y$ appartient à $F^{-1}\left(V_{n-1}^{\prime}\right)$ et, $P_{n-1}$ étant un voisinage de $F^{-1}\left(V_{n-1}^{\prime}\right), y(p)$ appartient à $P_{n-1}$ pour tout $p$ assez grand. Cette contradiction prouve l'affirmation.

Comme $M_{n}$ contient $\bar{W} \cap H_{n}(X), N_{n}$ contient $X \cup\left(\bar{W} \cap G_{n}(X)\right)$, et la parfaite normalité de $E(X)$ nous permet de trouver un voisinage fermé $V_{n}$ de $X \cup\left(\bar{W} \cap G_{n}(X)\right)$ dans $G_{n}(X)$ contenu dans $N_{n}$ et vérifiant $V_{n} \cap G_{n-1}(X)=$ $V_{n-1}$ et $\operatorname{Int}_{n} V_{n} \cap G_{n-1}(X)=\operatorname{Int}_{n-1} V_{n-1}$. Soit $V_{n}^{\prime}$ un voisinage fermé de $V_{n}$ dans $G_{n}(X)$ contenu dans $N_{n}$.

D'après le lemme $5.4, H_{n}(Y)$ est métrisable; soit $d_{n}$ une distance admissible sur $H_{n}(Y)$. Quitte à remplacer $d_{n}$ par $d_{n}+d_{Y}$, nous pouvons supposer 
que

$$
d_{Y}\left(y, y^{\prime}\right) \leq d_{n}\left(y, y^{\prime}\right) \quad \forall y, y^{\prime} \in H_{n}(Y) .
$$

Les lemmes 5.2 et 5.3 entraînent que, pour tout $p \geq n, E_{p+1}(Y) \cap H_{n}(Y)$ est un voisinage de $E_{p}(Y) \cap H_{n}(Y)$ dans $H_{n}(Y)$. Nous pouvons alors trouver une fonction continue $\left.\left.\eta_{n}: H_{n}(Y) \rightarrow\right] 0,1\right]$ vérifiant

$$
\text { pour tout } p \geq n \text { et tout point } y \text { de } E_{p}(Y) \cap H_{n}(Y) \text {, }
$$

$$
\eta_{n}(y)<d_{n}\left(y, H_{n}(Y) \backslash E_{p+1}(Y)\right) .
$$

Définissons une fonction continue $\widetilde{\varphi}_{n}: F^{-1}\left(M_{n}\right) \rightarrow 2^{H_{n}(Y)}$ par $\widetilde{\varphi}_{n}(y)=$ $\varphi_{n}(F(y))$. Comme $F^{-1}\left(M_{n} \backslash \bar{W}\right)$ est ouvert dans $F^{-1}\left(G_{n}(X)\right)$, qui est fermé dans $E(Y)$, c'est, d'après le lemme 2.3, une réunion dénombrable de compacts de dimension finie, et il en est de même de tout espace $\left(F^{-1}\left(M_{n}\right)\right.$ $\bar{W}), \tau)$ où $\tau \in \mathcal{T}(Y)$. Appliquant à nouveau le lemme 5.1 à un espace $\left(F^{-1}\left(M_{n} \backslash \bar{W}\right), \tau\right)$ où $\tau$ est judicieusement choisie, nous pouvons trouver une fonction continue $\chi_{n}: F^{-1}\left(M_{n} \backslash \bar{W}\right) \rightarrow H_{n}(Y)$ vérifiant, pour tout $y \in F^{-1}\left(M_{n} \backslash \bar{W}\right)$,

$$
\begin{aligned}
d_{n}\left(\chi_{n}(y), \tilde{\varphi}_{n}(y)\right) & <\min \left(d_{n}\left(\widetilde{\varphi}_{n}(y), H_{n}(Y) \backslash P_{n-1}\right)\right. \\
& \left.d_{Y}\left(y, F^{-1}\left(G_{n-1}(X) \cup \bar{W}\right)\right), \min \left\{\eta_{n}(z): z \in \widetilde{\varphi}_{n}(y)\right\}\right) .
\end{aligned}
$$

Ici encore, la relation (9) garantit que $\chi_{n}(y)$ appartient à $P_{n-1}$, mais nous ne pouvons pas définir $k_{n}$ de la même façon que $k_{1}$ car $k_{n-1}$ ne se factorise pas à travers $F$. Il nous faut donc modifier un peu la suite de la construction. Si $y, y^{\prime}$ sont deux points de $E(Y)$, nous notons $\left[y, y^{\prime}\right]$ le segment de droite d'extrémités $y$ et $y^{\prime}$. Posons

$$
\begin{aligned}
R_{n} & =\left\{y \in F^{-1}\left(M_{n} \backslash \bar{W}\right):\left[y, \chi_{n}(y)\right] \subset P_{n-1}\right\}, \\
T_{n} & =R_{n} \cup F^{-1}\left(\operatorname{Int}_{n-1} V_{n-1}^{\prime} \cup \bar{W}\right) .
\end{aligned}
$$

AfFirmation 2. $T_{n}$ est ouvert dans $F^{-1}\left(G_{n}(X) \cup \bar{W}\right)$.

Comme $M_{n}$ et $P_{n-1}$ sont ouverts et la fonction $\chi_{n}$ continue, $R_{n}$ est ouvert dans $F^{-1}\left(G_{n}(X) \cup \bar{W}\right)$. En raisonnant comme dans la démonstration de l'affirmation 1, on constate que, si $T_{n}$ n'etait pas ouvert, il existerait un entier $q \geq n$ et des points $z \in E_{q}(Y) \cap F^{-1}\left(\operatorname{Int}_{n-1} V_{n-1}^{\prime} \cup \bar{W}\right)$ et $z(p) \in E_{q}(Y) \cap$ $\left(F^{-1}\left(G_{n}(X) \cup \bar{W}\right) \backslash T_{n}\right), p \geq 1$, tels que la suite $\{z(p)\}_{p=1}^{\infty}$ converge vers $z$. D'après l'affirmation $1, N_{n} \cup \bar{W}$ est un voisinage de $\operatorname{Int}_{n-1} V_{n-1}^{\prime} \cup \bar{W}$ dans $G_{n}(X) \cup \bar{W}$, donc nous pouvons supposer que, pour tout $p, z(p) \in F^{-1}\left(N_{n} \cup\right.$ $\bar{W}) \backslash T_{n}=F^{-1}\left(M_{n} \backslash \bar{W}\right) \backslash R_{n}$, donc le segment $\left[z(p), \chi_{n}(z(p))\right]$ n'est pas entièrement contenu dans $P_{n-1}$. Puisque $z(p)$ appartient à $E_{q}(Y), F(z(p))$ appartient à $E_{q}(X)$ et le lemme 5.2 entraîne que $\widetilde{\varphi}_{n}(z(p))=\varphi_{n}(F(z(p)))$ est contenu dans $E_{q}(Y)$. Alors, les conditions (8) et (9) entraînent que $\chi_{n}(z(p))$ appartient à $E_{q+1}(Y)$. Comme $E_{q+1}(Y)$ est un compact métrisable, nous 
pouvons, quitte à extraire une sous-suite, supposer que $\left\{\chi_{n}(z(p))\right\}$ converge vers un point $a$ et que le suite de compacts $\left\{\widetilde{\varphi}_{n}(z(p))\right\}$ converge vers un compact $K$. Puisque $F\left(\widetilde{\varphi}_{n}(z(p))\right)=F(z(p)), K$ est contenu dans $F^{-1}(F(z))$. Comme $\left\{d_{Y}\left(z(p), F^{-1}\left(G_{n-1}(X) \cup \bar{W}\right)\right)\right\}$ tend vers zéro, il résulte de (9) et (7) que

$$
d_{Y}\left(\chi_{n}(z(p)), \widetilde{\varphi}_{n}(z(p))\right) \rightarrow 0 .
$$

Comme $E_{q+1}(Y)$ est compact, la distance continue $d_{Y}$ définit sa topologie, donc (10) entraîne que la limite $a$ de $\left\{\chi_{n}(z(p))\right\}$ appartient à $K$, donc que $F(a)=F(z)$. Mais alors, $[z, a] \subset F^{-1}(F(z)) \subset F^{-1}\left(V_{n-1}^{\prime} \cup \bar{W}\right) \subset P_{n-1}$. Puisque $\left\{\chi_{n}(z(p))\right\}$ tend vers $a$, le segment $\left[z(p), \chi_{n}(z(p))\right]$ tend vers $[z, a]$, donc, $P_{n-1}$ étant ouvert, est entièrement contenu dedans si $p$ est assez grand. Cette contradiction prouve l'affirmation.

Comme $T_{n}$ contient le fermé $F^{-1}\left(\left(V_{n}^{\prime} \cap G_{n-1}(X)\right) \cup \bar{W}\right)$, nous pouvons trouver un ouvert $B$ de $F^{-1}\left(G_{n}(X) \cup \bar{W}\right)$ vérifiant $F^{-1}\left(\left(V_{n}^{\prime} \cap G_{n-1}(X)\right) \cup\right.$ $\bar{W}) \subset B \subset \bar{B} \subset T_{n}$. Soit $\beta$ une fonction continue de $F^{-1}\left(G_{n}(X) \cup \bar{W}\right)$ dans $I$ telle que $\beta(y)=0$ si $y \in \bar{B}$ et $\beta(y)=1$ si $y \notin T_{n}$. D'après la définition de $T_{n}$, le point $(1-\beta(y)) y+\beta(y) \chi_{n}(y)$ appartient à $P_{n-1}$ quel que soit $y \in F^{-1}\left(M_{n} \backslash \bar{W}\right)$, ce qui nous permet de définir une fonction continue $k_{n}: F^{-1}\left(V_{n}^{\prime} \cup \bar{W}\right) \rightarrow S^{m}$ par

$$
k_{n}(y)= \begin{cases}\bar{k}_{n-1}(y) & \text { si } y \in \bar{B} \\ \bar{k}_{n-1}((1-\beta(y)) y+\beta(y) \chi(y)) & \text { si } y \notin B .\end{cases}
$$

Puisque $\bar{k}_{n-1}$ prolonge $k_{n-1}, k_{n}$ vérifie (4) et (5). Ceci achève la démonstration du lemme 4.1.

\section{Bibliographie}

[1] V. N. Basmanov, Foncteurs covariants, rétractes et dimension, Dokl. Akad. Nauk SSSR 271 (1983), 1033-1036 (en russe).

[2] J. van der Bijl and J. van Mill, Linear spaces, absolute retracts, and the compact extension property, Proc. Amer. Math. Soc. 104 (1988), 942-952.

[3] R. Cauty, Une caractérisation des rétractes absolus de voisinage, Fund. Math. 144 (1994), 11-22.

[4] T. Dobrowolski, On extending mappings into nonlocally convex linear metric spaces, Proc. Amer. Math. Soc. 93 (1985), 555-560.

[5] A. N. Dranishnikov, Sur un problème de P. S. Aleksandrov, Mat. Sb. 135 (177) (1988), 551-557 (en russe).

[6] R. Engelking, General Topology, PWN, Warszawa, 1977.

[7] -, Dimension Theory, PWN, Warszawa, 1978.

[8] W. E. Haver, Locally contractible spaces that are absolute neighborhood retracts, Proc. Amer. Math. Soc. 40 (1973), 280-284.

[9] -, A near-selection theorem, General Topology Appl. 9 (1978), 117-124. 
[10] J. L. Kelley and I. Namioka, Linear Topological Spaces, van Nostrand, New York, 1963.

[11] V. Klee, Leray-Schauder theory without local convexity, Math. Ann. 141 (1960), $286-296$.

[12] G. Kozlowski, Factorization of certain maps up to homotopy, Proc. Amer. Math. Soc. 21 (1969), 88-92.

[13] J. J. Walsh, Isotoping mappings to open mappings, Trans. Amer. Math. Soc. 250 (1979), 121-145.

22, RUE JOUVENET

F-75016 PARIS, FRANCE

Received 26 October 1993;

in revised form 12 May 1994 